\title{
PROPRIEDADES FÍSICO-QUÍMICAS DO AMIDO DO CARÁ (Dioscorea cayennensis) NATIVO E MODIFICADO POR ACETILAÇÃO
}

\section{PHYSICAL-CHEMICAL PROPERTIES OF CARARCH STARCH (Dioscorea cayennensis) NATIVE AND MODIFIED BY ACETATION}

\author{
M. C. S. OLIVEIRA ${ }^{1}$, J. H. JESUS ${ }^{1 *}$, F. M. M. BRONDANI ${ }^{1}$, R.R.O. LIMA ${ }^{1}$, R. VIEIRA ${ }^{2}$.
}

${ }^{1}$ Faculdade de Educação e Meio Ambiente - FAEMA, Ariquemes, RO, Brasil

${ }^{2}$ Instituto Federal de Rondônia, IFRO, Ji Paraná, RO, Brasil

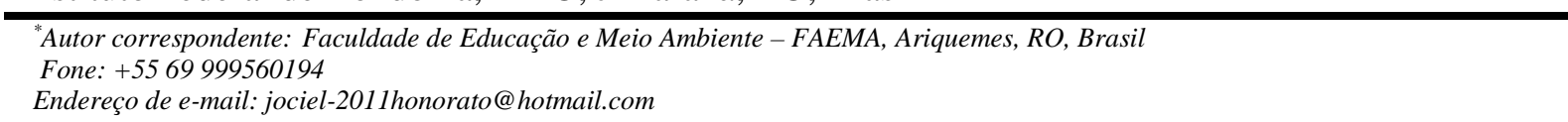

\begin{tabular}{l} 
A R T I C L E I N F O \\
\hline Article history: \\
Received 2018-09-06 \\
Accepted 2018-10-14 \\
Available online 2018-10-31 \\
pa lavras-chave \\
Amido \\
Modificação \\
Acetilação \\
$\frac{k e y w o r d s}{\text { Starch }}$ \\
Modification \\
Acetylation
\end{tabular}

\begin{abstract}
R E S U M O
O Cará é uma planta de raiz tuberosa, longas e achatadas com formato de uma moela, os bulbos aéreos. Pertence à família Dioscoriaceae Dicotyledonea e ao gênero Dioscorea com variedades de espécies e alguns são utilizados como alimentos. Esta pesquisa foi desenvolvida com o objetivo de estudar as propriedades funcionais do amido nativo e modificado através da acetilação. De $3,750 \mathrm{~kg}$ do Cará foram extraídos $287 \mathrm{~g}$ de amido. As análises da forma e tamanho de grânulos do amido nativo e modificado, a microscopia óptica, com visualização nas objetivas de 40X e 10X. a solubilidade e poder de intumescimento foram determinados em diferentes temperaturas e $\mathrm{pH}$. A capacidade de absorção de água e óleo do método descrito por Okezie e Bello. A claridade da pasta foi quantificada como descrito por Demiate sendo empregada a pasta de $1 \%$. A solubilidade dos amidos teve variação de $6.66 \%$ até $60 \%$ entre o nativo e o modificado em função da temperatura. Em função do $\mathrm{pH}$ há uma variação de $7 \%$ a $40 \%$ quanto ao poder de intumescimento. A capacidade do amido modificado de absorver mais água e óleo que o nativo demonstra que torna um produto apto para ser utilizado nas indústrias principalmente alimentícia na qual se faz necessário reduz o teor de lipídeos dos alimentos. A pasta do amido nativo sofre uma retrogradação maior após alguns dias, pois seu amido tem uma mudança natural em sua estrutura.
\end{abstract}

\section{A B S T R A C T}

The Cará is a tuberous root plant, long and flat shaped like a gizzard, the aerial bulbs. It belongs to the Dioscoriaceae family Dicotyledonea and to the genus Dioscorea with varieties of species and some are used as food. This research was developed with the objective of studying the functional properties of native and modified starch through acetylation. Of 3,750 $\mathrm{kg}$ of Cará, $287 \mathrm{~g}$ of starch was extracted. Analyzes of the shape and size of native and modified starch granules, optical microscopy, with visualization in the 40X and 10X objectives. The solubility and swelling power were determined at different temperatures and $\mathrm{pH}$. The water and oil absorption capacity of the method described by Okezie and Bello. The clarity of the slurry was quantified as described by Demiate using the $1 \%$ paste.The solubility of starches varied from $6.66 \%$ to $60 \%$ between native and modified as a function of temperature. Depending on the $\mathrm{pH}$ there is a variation of $7 \%$ to $40 \%$ in the swelling power. The ability of the modified starch to absorb more water and oil than the native one demonstrates that it makes a product suitable for use in the mainly food industries in which it is necessary to reduce the lipid content of foods. The starch paste native undergoes a greater retrogradation after a few days, as its starch has a natural change in its structure. 
Dentre as variedades de raízes tuberosas no mundo alimentício destacam-se a mandioca, a batata, a batata-doce e também o cará pertencente à família das Dioscoreas. Conforme Ferreira (2011), as Dioscoreáceas compõem uma fonte alimentar importantes e estão distribuídas nas regiões tropicais, subtropicais e temperadas de todo mundo.

Uma nutrição inspirada na cultura regional, torna-se uma opção eficaz no combate à insegurança alimentar, portanto, mantimentos da região como frutas, hortaliças, tubérculos e leguminosas são aqueles disponíveis em cada região do Brasil e possuem como característica primordiais o fácil acesso, o baixo custo e o alto valor nutritivo. (MARTINS, et al. 2015 e BRASIL, 2002).

De acordo com o clima de Rondônia sendo tropical, quente e úmido, torna-se um local favorável para o cultivo das espécies acima citadas. Como o "Cará" é um tubérculo típico nessa região do norte do país e suas propriedades são pouco conhecidas pela população consumidora, isso faz com que seu consumo ainda seja limitado. (SCLINDWEIN, et al. 2012)

Há possibilidade de aumento da exploração desse cultivo, com incentivo na plantação e custos econômicos aceitáveis para os agricultores, havendo a necessidade de estruturar a cadeia produtiva, fortalecer os atuais sistemas de plantio, potencializar o modo das indicações e métodos disponíveis. (SANTOS et al. 2007).

Os mesmos autores mencionam que os tubérculos de cará possuem características nutritivas e energéticas relevantes, sendo abastados em vitaminas do complexo B (tiamina, riboflavina, niacina, adermina), carboidratos, principalmente amido, minerais e propriedades medicinais, além de exibirem baixo teor de gordura.

O amido é o carboidrato fundamental consumido pelo homem e proporciona numerosos aproveitamentos nas indústrias. Os amidos nativos têm uso limitado na indústria alimentícia devido à sua fraca estabilização térmica e ácida, bem como muita facilidade a retrogradação. No qual podem ser alterados, visando contrair maior permanência e melhorias nas propriedades reológicas das pastas, textura dos géis e retenção de água. (LIMA; SILVA JUNIOR; DE SÁ (2014)

A alteração na fonte de amido, composição e arranjo, bem como as distinções nas propriedades, torna o amido apropriado a uma variedade de aplicações fornecida para diversas funcionalidades, como por exemplo, nas indústrias alimentícias. (SANTOS, 2016).

Os métodos e reagentes empregados na manufatura, tanto dos amidos modificados, quanto nos amidos já industrializados, tem sido intensamente averiguado e testado para enriquecimento de sua funcionalidade. No entanto o uso de amidos modificados vem auferindo seriedade contínua na indústria alimentícia, pois o amido pode ser alterado quimicamente e fisicamente para atender solicitações especiais. (PEREIRA, 2011)

Desse modo, realizar um estudo exploratório da riqueza estrutural do amido do cará, visando sua modificação através de uma reação de acetilação, traz à sociedade conhecimentos de um alimento muito conhecido nesta região, com grande potencial alimentício e até mesmo nos comércios para a exportação trazendo lucros financeiros.

\section{MATERIAL E MÉTODOS}

\subsection{EXTRAÇÃO E MODIFICAÇÃO DO AMIDO DO CARÁ}

O Cará (Dioscorea cayennensis) foi colhido em um sítio na região da cidade de Buritis, localizado a $125 \mathrm{~km}$ do município de Ariquemes, estado de Rondônia. As análises laboratoriais foram realizadas nos laboratórios de Bromatologia, Farmacotécnica e Química Geral da Faculdade de Educação e Meio Ambiente - FAEMA, localizada na avenida machadinho, setor 06 Ariquemes Rondônia.

O pré-processo iniciou-se pesando 3,750 kg do Cará, em seguida foi descascado, picado e triturado no liquidificador, utilizando 4 litros de água destilada e, sequencialmente, tamisado com peneira de 0,42 mesh, após acrescentado 7 litros de água destilada e peneirado numa abertura de 0,25 mesh. Seu ciclo de decantação ocorre por volta de $24 \mathrm{hs}$, para cada lavagem, sendo peneirado em abertura de 0,25 mesh e 0,18 mesh, para a obtenção de uma massa branca e água clara descartando o sobrenadante. A seguir, foi seco em estufa de secagem com circulação de ar marca: Nova Ética; modelo: 400/2ND-300 por 48hs, e tamisado numa peneira de 2,00 mesh.

Foi pesado $100 \mathrm{~g}$ do amido, adicionado $500 \mathrm{~mL}$ de água e agitado em chapa aquecedora com agitador magnético de marca Biomax com $n^{\circ}$. de série 13800 por 20 minutos. Para a modificação o método utilizado foi adaptado por Sathe; Salunke (1981).

Com pHmetro calibrado em temperatura ambiente o $\mathrm{pH}$ foi ajustado entre 8,0 e 8,5. Utilizou-se solução de hidróxido de sódio $1 \mathrm{~mol} / \mathrm{L}, 20,4 \mathrm{~g}$ de anidrido acético, deixando a reação ocorrer por mais cinco minutos. Em seguida, usando ácido clorídrico 0,5 mol/L, ajustou-se o $\mathrm{pH}$ para faixa de 4,0 e 4,5. Em seguida a amostra foi filtrada a vácuo, em funil de Büchner e lavada com água destilada. Sequencialmente foi levada para secar por $24 \mathrm{hs}$ na estufa com circulação de ara $34^{\circ} \mathrm{C}$. O amido modificado foi peneirado em peneira de 0,18 mesh e guardado em tempo de conservação.

\subsection{MICROSCOPIA ÓTICA}

Realizado em triplicata, utilizando microscópio óptico de marca OPTON (modelo N-101 B) visualizando as objetivas de 40x e 10x, analisou o tamanho e a forma dos grânulos do amido nativo e modificado, preparando $100 \mathrm{mg}$ de amido em $5 \mathrm{~mL}$ de solução 1:1 de glicerina: água, colocando duas gotas em uma lâmina e cobrindo com uma lamínula.

\subsection{PROPRIEDADES FUNCIONAIS DO AMIDO NATIVO E MODIFICADO}

\subsubsection{Solubilidade e poder de Intumescimento em função da temperatura}

Utilizando o método adaptado por Leach; McCowen; Schoch (1959), preparou-se uma solução de $0,1 \mathrm{~g}$ de amostra em $10 \mathrm{~mL}$ de água destilada e, em seguida foi colocada em banhomaria nas temperaturas de $55^{\circ}, 65^{\circ}, 75^{\circ}, 85^{\circ}$ e $95 \mathrm{C}^{\circ}$, mantidos em agitação por 30 minutos. Em seguida, os tubos foram levados a centrífuga e centrifugados a $1000 \mathrm{rpm}$ por 15 minutos. Descartado o sobrenadante, foram coletados para a quantificação da fração solúvel em tubos e pesados. A solubilidade é expressa em grama por $100 \mathrm{~g}$ em base seca, 
enquanto o poder de intumescimento é a relação da massa final pela inicial, onde as análises foram realizadas em triplicata.

Realizada em determinadas temperaturas as quais descritas acima, para promover a quebra das pontes de hidrogênio deixando livres grupos de hidroxila, permitindo que os grânulos continuem a inchar e resultem no aumento da solubilidade do amido.

\subsubsection{Solubilidade e poder do intumescimento em função do pH}

Para estudos do $\mathrm{pH}$ relacionado ao amido nativo e modificado quanto se solubiliza e quanto influência no inchamento dos grânulos, foi preparado solução de ácido clorídrico a $0,1 \mathrm{M} / \mathrm{L}$ e hidróxido de sódio a $0,12 \mathrm{M} / \mathrm{L}$ com água destilada. As amostras utilizadas foram de $1 \% \mathrm{p} / \mathrm{v}$, ajustando o $\mathrm{pH}$ para os valores almejados de 4, 6, 8, 10 e 12, colocadas na estufa por $1 \mathrm{~h}$, a $30^{\circ} \mathrm{C}$, e em seguida, centrifugadas a $1000 \mathrm{rpm}$ por 15 minutos.

\subsubsection{Capacidade de absorção de água e óleo}

Na observação da capacidade de absorção de água ou de óleo foi empregado o método descrito por Okezie; Bello (1988) no qual a amostra de $1 \mathrm{~g}$ de amido nativo e modificado foram solubilizadas em $50 \mathrm{~mL}$ de água e as mesmas quantidades de amostras em $50 \mathrm{~mL}$ de óleo, foram preparadas as soluções e colocadas em tubos de ensaio, agitada em agitador votex para tubos marca Quimis modelo Q220 por 1minuto e sequencialmente levados à centrifugada para tubos, marca Quimis; modelo:Q222T216 a 1500 rpm por 20 minutos. Medindo o peso da amostra antes e depois da absorção. A capacidade de absorção é relacionada com a quantidade de água absorvida em $100 \mathrm{~g}$ de amostra, a análise foi realizada em triplicata.

\subsubsection{Claridade da pasta e poder de retrogradação}

O método usado para a claridade da pasta e poder de retrogradação foi descrito por Demiate; Kotovicz (2011). Quantificando que uma solução de $1 \%$ de amido é aquecida a $98^{\circ} \mathrm{C}$ a banho-maria por 30minutos para promover a gelatinização completa dos grânulos, com pouca agitação. Em seguida o amido foi resfriado e a solução foi analisada no espectrofotômetro (marca Quimis, modelo Q798DP) a 680nm. O procedimento foi feito em triplicata.

\section{RESULTADOS E DISCUSSÃO}

$\mathrm{Na}$ extração do amido foram obtidos $287 \mathrm{~g}$ de amido nativo, porém utilizou $100 \mathrm{~g}$ para a realização de modificação. $\mathrm{Na}$ realização da análise da microscopia ótica a glicerina dificulta a absorção de água pelos grânulos impedindo de inchar e apresentar diâmetros maiores que os reais. Podendo ser observado o comportamento dos grânulos nas figuras 1 e 2 das amostras do amido modificado e da amostra do amido nativo, não houve mudanças de uma amostra de amido para a outra.
(A)

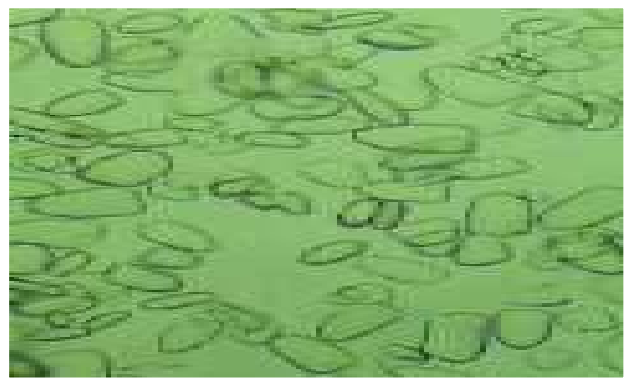

(B)

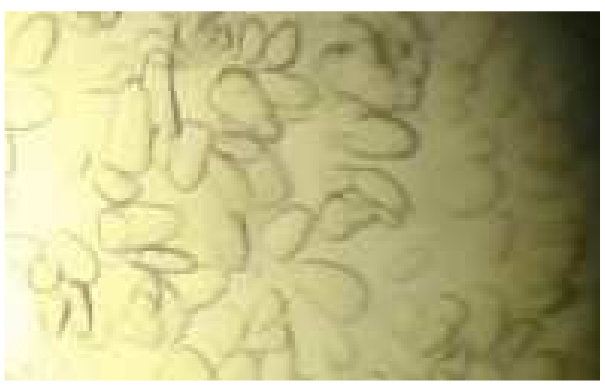

Figura 1- (A) grânulos de amidonativo, (B) grânulos de amido modificado ampliados em 40X

(C)

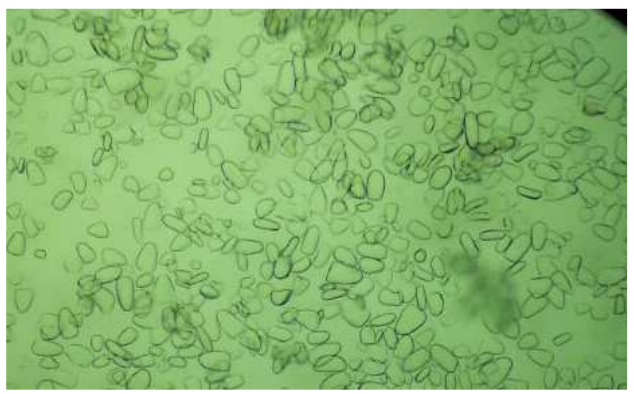

(D)

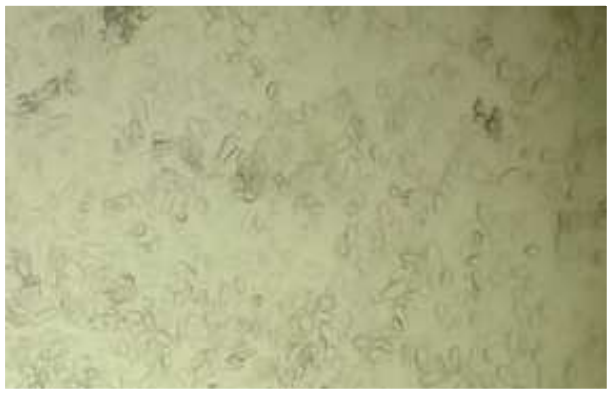

Figura 2 - (C) grânulos de amido nativo, (D) grânulos de amido modificado ampliados em 10X. 
Em semelhança da pesquisa com o feijão andú, de Lima; Silva Junior; De Sá (2014), descreveram que os grânulos eram ovalados e circulares, um pouco diferente ao encontrado nesta pesquisa, sendo ovalados e quadrado. O gráfico abaixo, retratado na figura 3 , apresenta os resultados obtidos no estudo comparativo entre os diferentes tipos de amido.

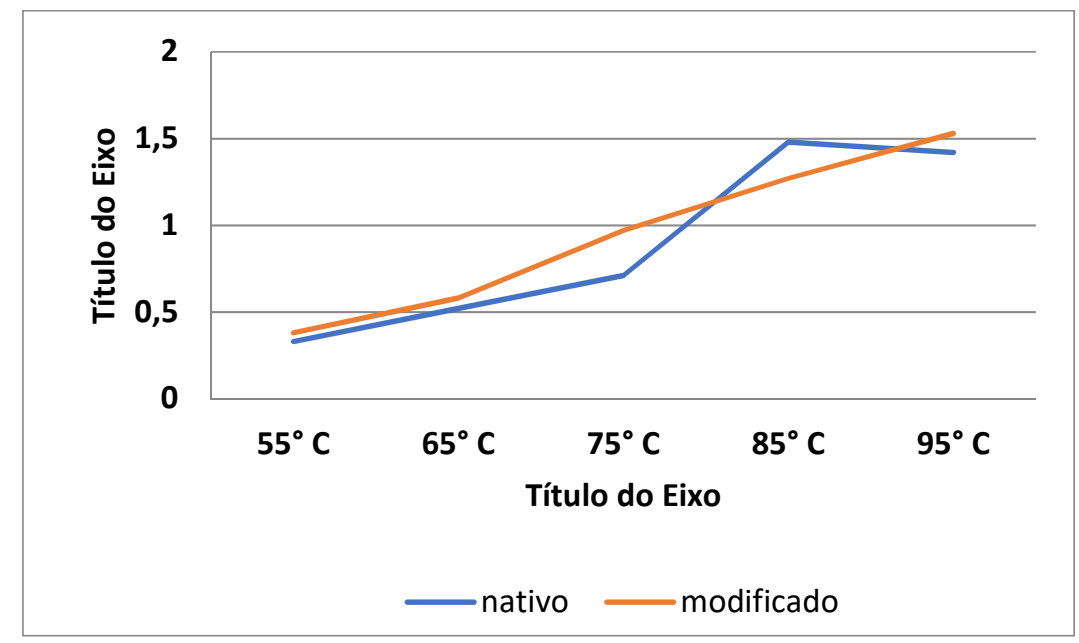

Figura 3 - Solubilidade dos amidos nativos e modificados do Cará cayemensis em função da temperatura

Em um estudo analítico do gráfico representado acima, verificou-se que tanto o amido nativo, quanto o modificado, na faixa de temperatura de $55^{\circ}$ até $65^{\circ}$ apresentam comportamento semelhantes, destacando-se o amido nativo que apresentou maior solubilidade nessa referida temperatura.

$\mathrm{Na}$ segunda faixa selecionada (de $65^{\circ}$ até $75^{\circ}$ ), nota-se que o amido nativo permaneceu em uma reta ascendente constante, porém, o amido modificado apresenta crescimento relevante na solubilidade, indo de $0,6 \mathrm{~g} / 100 \mathrm{~g}$ para $1,0 \mathrm{~g} / 100 \mathrm{~g}$, apresentando um aumento de $66,6 \%$ na solubilidade nesta faixa de temperatura.
Verificando a temperatura de $75^{\circ}$ até $85^{\circ}$ notou-se que essa faixa de temperatura foi possível verificar a maior discrepância entre os dois tipos de amido. Enquanto o amido modificado apresentou uma solubilização constante com o aumento da temperatura, o amido nativo apresentou uma elevação brusca dessa propriedade físico-química, aumentando de aproximadamente $0,7 \mathrm{~g} / 100 \mathrm{~g}$ para $1,5 \mathrm{~g} / 100 \mathrm{~g}$, correspondendo a mais que $100 \%$ de elevação da solubilidade.

E por fim, na última faixa analisada, de $85^{\circ}$ até $95^{\circ}$, o amido modificado permaneceu com aumento constante da solubilidade, enquanto que o amido nativo sofreu um decréscimo de $6,66 \%$.

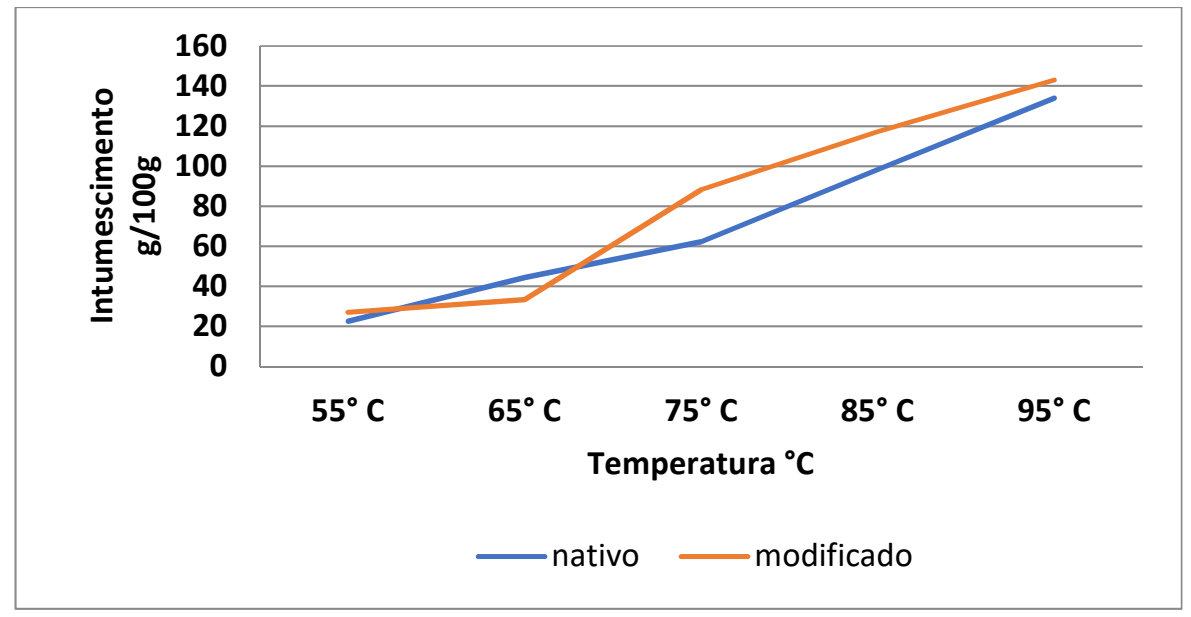

Figura 4- Poder de Intumescimento dos amidos nativos e modificados do cará Cayemensis em função da temperatura

Avaliando a figura 4 observa-se que o amido nativo devido suas propriedades reológicas que depende da temperatura e agitação para se solubilizar começa seu poder de intumescimento de forma gradativa acelerando o ritmo a partir da temperatura $75^{\circ} . \mathrm{O}$ amido modificado começa praticamente a partir da temperatura $65^{\circ}$ com rápida elevação em seu poder de intumescimento até a temperatura $75^{\circ}$. 


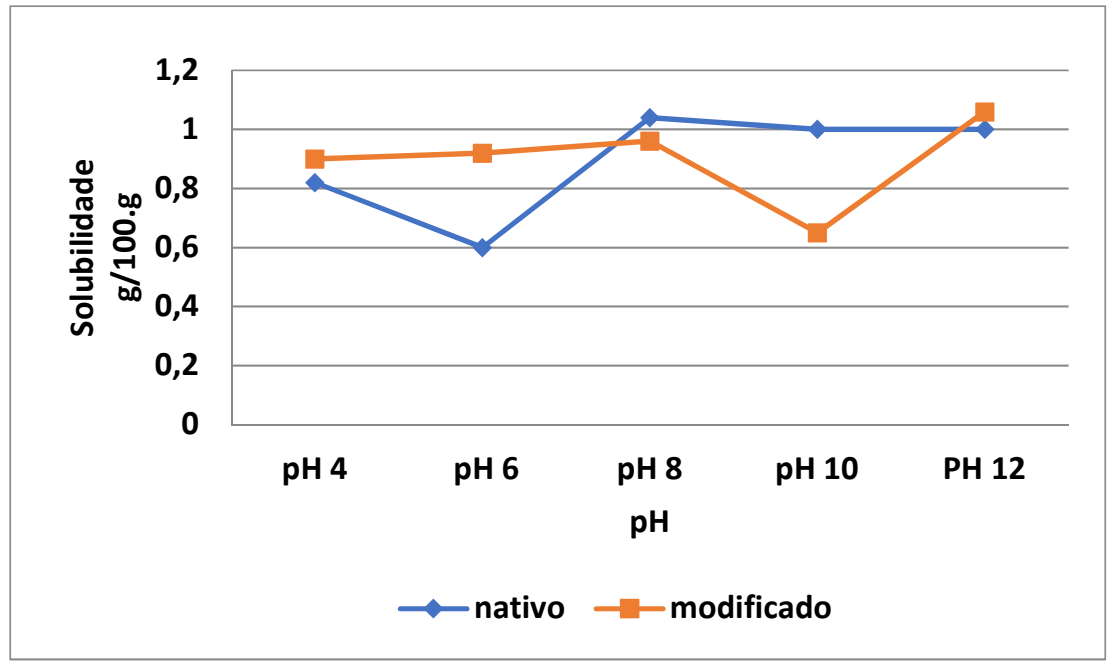

Figura 5 - Solubilidade dos amidos nativos e modificados do Cará Cayemensis em função do pH

Em análise do gráfico representado na figura 5, pode-se perceber que no $\mathrm{pH} 4$ o modificado solubilizou um pouco mais que o nativo, o qual no $\mathrm{pH} 6$ teve grande dificuldade para se solubilizar, enquanto o modificado manteve o mesmo comportamento no $\mathrm{pH} 4,6$ e 8. Porém, no $\mathrm{pH} 8$ o amido nativo teve grande facilidade de solubilização tendo uma dificuldade no $\mathrm{pH} 10$, mantendo um comportamento no $\mathrm{pH} 10$ e 12, diferentemente do modificado que teve grande dificuldade de solubilizar no $\mathrm{pH}$ 10, aumentando sua solubilidade em $\mathrm{pH}$ mais básico.

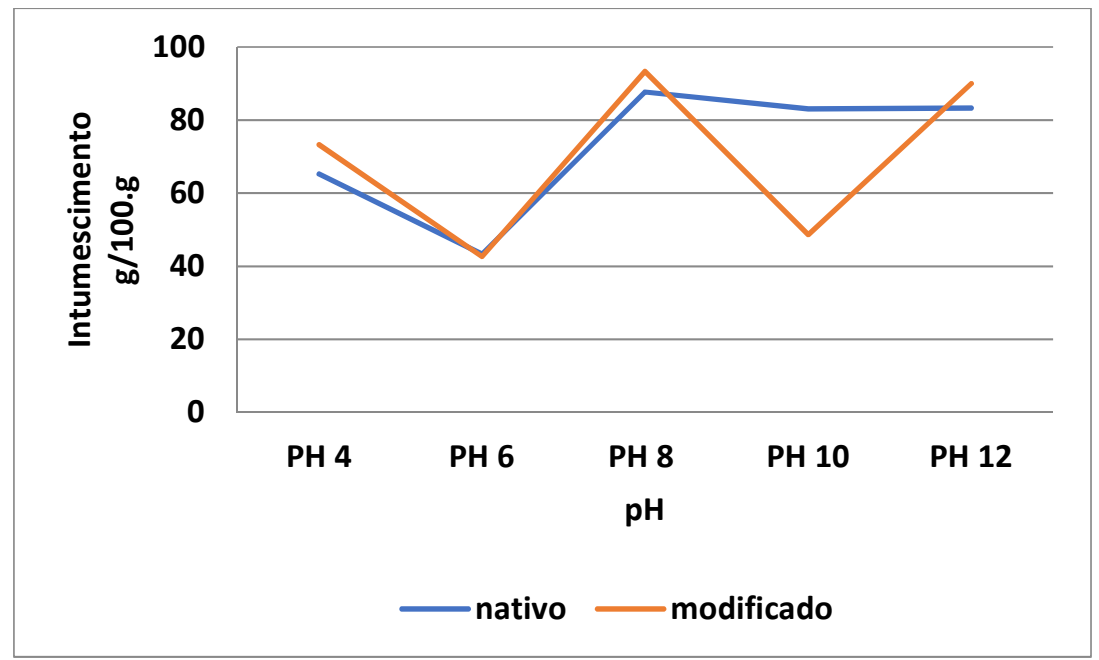

Figura 6 - Poder de Intumescimento em função do pH dos amidos nativo e modificado do Cará Cayemensis

Estudando a figura 6 que contempla um gráfico do poder de intumescimento em função do $\mathrm{pH}$, verifica-se que inicialmente no $\mathrm{pH} 4$ ambos se comportam de forma parecida. Porém no $\mathrm{pH} 8$ o modificado tem poder de intumescimento maior que o nativo, no qual tem um comportamento praticamente igual nos próximos, tendo uma leve queda no pH10 mantendo igual no $\mathrm{pH} 12$, diferente do amido modificado que no $\mathrm{pH} 10$ tem uma brusca dificuldade no poder de intumescimento, já no pH 12 supera o amido nativo. 


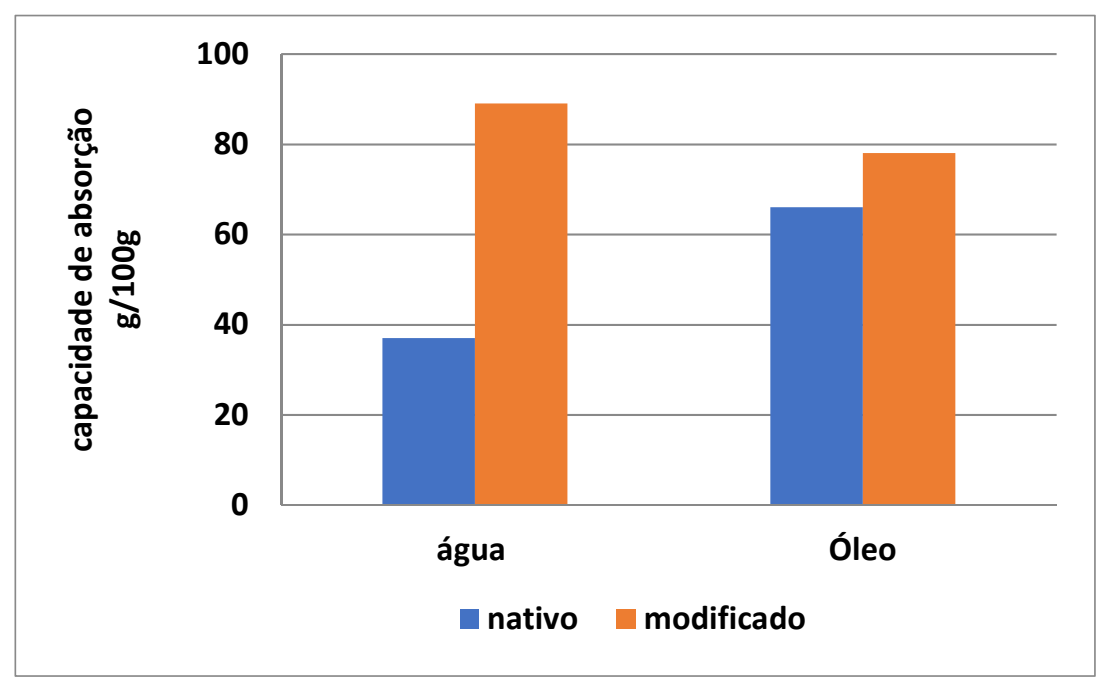

Figura 7 - Capacidade de absorção de água (CAA) e óleo (CAO) do amido nativo e modificado do Cará Cayemensis

O amido nativo devido sua dificuldade de solubilidade tem menor absorção de água quando comparado ao modificado, porém o amido modificado absorve menos óleo que o nativo, demonstrando a importância da modificação do amido como demonstrado em estudos de
Silva et al. (2006), pois o amido modificado reduz o teor de lipídeos nos produtos como maionese, produtos embutidos, etc.

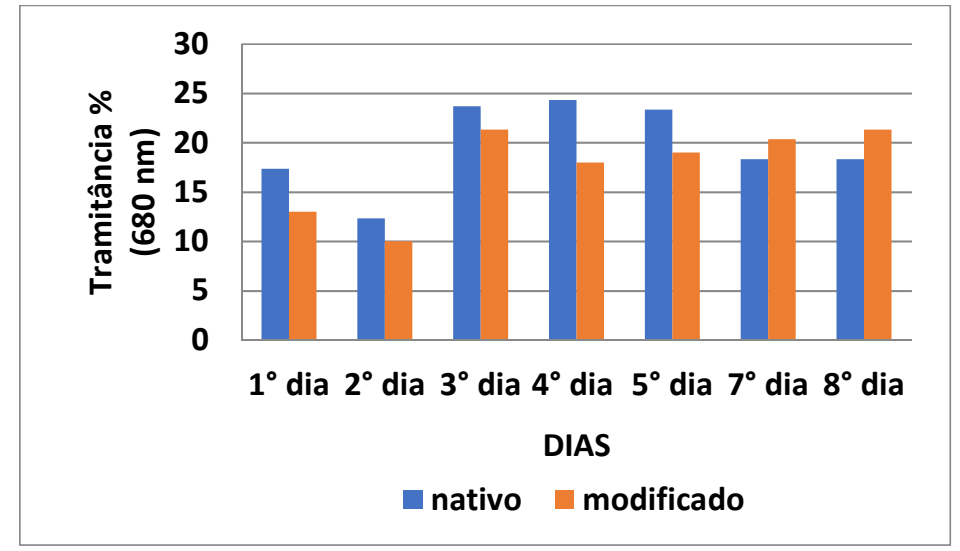

Figura 8 - Claridade da pasta e poder de retrogradação dos amidos nativos e modificados do Cará Cayemensis.

A pasta do amido nativo demonstrou que sua claridade nos primeiros dias são maiores que o modificado, porém conforme passam os dias o poder de retrogradação do amido modificado é maior, pois o amido nativo tem maior facilidade a regredir devido a sua modificação em sua estrutura.

\section{CONCLUSÕES}

Nos últimos anos o mercado de amido vem crescendo e se aperfeiçoando cada vez mais, levando à busca de produtos com características específicas que atendam as exigências industriais, diante disso, essas exigências podem ser solucionados com a modificação química, física ou enzimática do amido, tendo grandes possibilidades de introduzir novas matérias-primas agrícolas como fontes de amidos com características interessantes industrialmente, pois proporcionaria um crescimento diferenciado em nível mundial,
Por já ter havido a substituição dos grupamentos hidroxílicos, que são bastante polares e permitem interação de ligação de hidrogênio, por grupos mais volumosos, como os carboxílicos, que não fazem esse tipo de ligação intermolecular, a temperatura e $\mathrm{pH}$ é um fator determinante que pode ser atribuído a tais diferenças nas propriedades físico-químicas comparadas nesse estudo.

visto que no Brasil existe uma grande variedade de raízes amiláceas ainda pouco exploradas.

A extração do amido do Cará Dioscorea cayennensis foi com intuito de modificá-lo, para fazer um estudo comparativo dos resultados, visando identificar qual dos amidos, o nativo ou modificado, tem maior durabilidade, qual apresenta maior absortividade de água ou o óleo, quanto à solubilidade diante da temperatura e $\mathrm{PH}$.

Sendo possível observar que o amido modificado tem maior facilidade de absorção da água, com maior facilidade de 
solubilizar, pois o amido nativo não é facilmente solúvel em meio aquoso e absorve mais óleo. Sendo assim o amido modificado tem maior durabilidade que o nativo, pois seu poder

\section{R E F E R Ê N C I A S}

ABRAMO, M. A. Taioba, Cará e inhame: o grande potencial inexplorado. Editor Ícone, pág. 80. São Paulo, 1990.

APLEVICZ, K. S.; DEMIATE, I. M. Caracterização de amidos de mandioca nativos e modificados e utilização em produtos pacificados. Resv. Ciên. Tec. Alimen., vol.27, n³. Campinas- SP, 2007 Disponível em: http://www.scielo.br/scielo.php?script=sci_arttext\&pid= S0101-20612007000300009.

BRASIL. Alimentos Regionais Brasileiros. Ministério da Saúde; 2002. Disponível em: http://189.28.128.100/ nutrição/docs/geral/alimentos_regionais_brasileiros.pdf

CAUVAIN, S. P.; YOUNG, L. S. Tecnologia da panificação, editora Manole, $2^{\circ}$ ed., São Paulo, 2009.

DEMIATE, I. M.; KOTOVICZ, V. Amido de mandioca na indústria Brasileira de Alimentos. Ciência e Tecnologia de Alimentos, vol. 31, $\mathrm{n}^{\circ} 2$, pág. 388-397, 2011. Disponível em: http://www. scielo.br/ scielo.php?script $=$ sci_arttext\&pid $=$ S.

DESHPANDE, S. S.; SATHE, S. K.; CORNFORTH, D.; SALUNKHE, D. K. Effects of Dehulling on functional properties of Dry Bean (phaseolus vugaris L.) Flours. Cereal Chemistry, v. 59, n. 5, 1982. Disponível em <http://www.aaccnet.org/publications/cc/backissues/198 2/Documents/chem59_396.pdf.

FERREIRA, A. B. Sistema de cultivo do cará Dioscorea SPP. Por pequenos agricultores da baixada cuiabana - MT. Júlio Mesquita Filho-UNESP, Botucatu-SP, 2011. Disponível em: /1http://repositorio.unesp.br /bitstream/handle/11449/93493/ferreira_ab_me_botfca. pdf? sequence=1\&isAllowed=y acesso em:14 set. 2016

FRENCH, D. Chemical and physical properties of starch. Journal of Animal Sciene, vol. 37, n²4, pág. 1048-1061, 1973.

FRENCH, D. Organization of stach granules.Starch: Chemistry and Technology, Academic Press, $2^{\circ} \mathrm{ed}$., pág. 183-247, London, 1984

HARRIS, D. C. Química Analítica Quantitativa. 5 ed. LTCLivros Técnicos e Científicos. RJ, 201.

HEREDIA, Z. N. A.; VIEIRA, M. C. Produção de Dois Clones de Cará Dioscorea sp. Considerando Três Populações, em Dourados-MS. SOBInforma, v. 13, n² 2, pag.24-26, Paraná, 1994.

HEREDIA, Z. N. A.; VIEIRA, M. C.; MINUZZI, A. Produção de Cará (Dioscorea Sp.) em Diferentes Densidades de Plantio. Cienc. Agrotec. Editora UFLA. pág 337. Dourados-MS, 2000. Disponível em: http://www.revistas.ufg.br/pat/article/view.

HEREDIA, Z. N. A.; VIEIRA, M. C.; ORTIZ, A. C. S. Produção de Clones de Cará em função de tipos de mudas. SOBInforma, v. 17, $\mathrm{n}^{\circ} 1$, pág.16-17. Rio de Janeiro, $1998 . \quad$ Disponíveis em: http://www.revistas.ufg.br/pat/article/view/2991/3034. de retrogradação demonstrou menor que a do nativo, podendo ser empregado em produtos que necessitam ficar mais tempo estocado.

LAWAL, O. S. Composition, phisicochemical propertis andregration, characteristcs of native, oxidized, acetylated and acid-thinned new cocoyam (Xanthosoma sagittifolium) starch. Food Chemistry, v.87, p. 205-218, 2004. Disponivel em: http:// www.sciencedirect. com/science/article/pii/S0308814603006162.

LEACH, H.W.; McCOWEN, L.D.; SCHOCH, T.J. Structure of the starch granule. I. Swelling and solubility patterns of various starches. Cereal Chemistry, v. 36, n. 6, p. 534$544,1959$.

LIMA, R. R. O.; SILVA JUNIOR, N.P.; DE SÁ, F. M.P. Propriedades Funcionais do Amido do Feijão Andú (Cajanus CajanL.) Nativo e Modificado por Acetilação. Revista científica da Faculdade de Educação e Meio Ambiente - FAEMA. 2014.

MARTINS, M. C.; FERREIRA, A.M.V.; NASCIMENTO, L. A.; AIRES, J. S.; ALMEIDA, P. C. de; XIMENES, L. B. Influência de uma estrategica educativa na promoção do uso de alimentos regionais. Revista RENE, UFC. Pag. 243, Ceará, 2015 Disponivel em: http://www. periodicos.ufc.br/index.php/rene/article/view/2718/2102 acesso em: 16/09/2016

MENDES, M. L. M. BORA, P. S. RIBEIRO, A. P. L. Propriedades morfológicas e funcionais e outras características da pasta do amido nativo e oxidado da amêndoa do caroço de manga ( Mangifera indica L.), variedade Tommy Atkyns. Instituto Adolfo Lutz, 71(1), 76-84. São Paulo, 2012. Disponível em: http://revistas.bvs-vet.org.br/rialutz/article/view/5351.

MUCCILLO, R. C. S. T. Caracterização e avaliação de Amido Nativo e Modificado de Pinhão Mediante Provas Funcionais e Térmicas. Lume-UFRS, Porto Alegre -RS, 2009. Disponível em: http://www.lume. ufrgs.br/handle/10183/18598.

OKEZIE, B.; BELLO, A. B. Physcochemical and functional properties of winged beans flour and isoled compares with soy isolated. Journal of Food Scienc., v. 53, p. 450, 1998.

OLIVEIRA, A. P.; FREITAS NETO, P. A.; SANTOS, E. S. Qualidade do inhame da Costa em função das épocas de colheita e da Adubação orgânica. Horticultura Brasileira, vol. 20, $\mathrm{n}^{\circ} 1$. Brasília-DF, 2002.

PARAIBA. OLIVEIRA A.P. Nutrição e época de colheita do inhame (Dioscorea $\mathrm{sp}$ ) e seus reflexos na produção e qualidade de rizóforos. In: Simpósio Nacional sobre as Culturas do Inhame e Taro, EMEPA-PB 1 vol. 2. pág. 83-98. Anais... João Pessoa, PB, 2002.

PARAIBA. SANTOS, E. S. Manejo sustentável da cultura do inhame (Dioscorea $s p$ ) no nordeste do Brasil. In Simpósio Nacional Sobre as Culturas de Inhame e Taro. EMEPA-PB, pág181-195. João Pessoa-PB, 2002. Disponível em: http:// gestão unificada.pb.gov.br/emepa/ publicacoes/revista-tca-emepa/ edições / volume-012007/volume-1- numero -1-setembro2007/tca06_inhame_prod.pdf . 
PEREIRA K. D. Amido resistente, a última geração no controle de energia e digestão saudável. Ciências e Tecnologia, vol. 27, Campinas-SP, 2007. Disponível em: http://www.scielo.br/pdf/cta/v27s1/a16v27s1.pdf visitado $17 / 09 / 2016$

PEREIRA, L. D. Caracterização do amido nativo e modificação química do amido da fruta- de - lobo (solanum lycocarpum) com tripolifosfato de sódio UEG, Goiás, 2011 disponível em: /3http:// repositorio. unesp.br/ bitstream/handle /11449/ 138153/ santos_ tpr_dr_ bot.pdf? sequence $=3$ \&isAllowed $=y$.

SANTOS, E., S., FILHO J., C.; LACERDA J., T.; CARVALHO R., A.Inhame (dioscorea sp.) Tecnologia de Produção e Preservação Ambiental. Tecnol. \& Ciên. Agropec. Vol 1. $\mathrm{N}^{\circ} 1$, pg 36, João Pessoa, 2007.

SANTOS, E. S. Inhame (Dioscorea spp.) aspectos básicos da cultura. EMEPA-PB, SEBRAE, pág158. João PessoaPB, 1996

SANTOS, E. S.; CAZÉ FILHO, J.; LACERDA, J.T. DE; CARVALHO, R. A. FONTÉLLI, I. S. C.; SILVA, J. B.; BARBOSA, M. M.; CASSIMIRO, C. M. Inhame e
Preservação Ambiental. EMBRAPA, EMEPA, pág.6. João Pessoa-PB, 2006

SANTOS, T. P. R. Efeito de modificação física sobre as propriedades de raízes das tuberosas. Júlio de Mesquita Filho-UNESP, São Paulo, 2016. Disponível em: http://www.pg.fca.unesp.br/Teses/PDFs/Arq1416.pdf.

SATHE, S. K.; SALUNKHE, D. K. Isolation, Partial Characterization and modification of the Grest Northern Bean (Phaseolus vulgaris L.) Journal of Food Science, vol. 46, p. 617-621, 1981.

SCHLINDWEIN, J. A.; MARCOLAN, A. L.; FIORELIPERIRA, E. C.; PEQUENO, P. L. L.; MILITÃO, J. S. T. L. 2012. Solos de Rondônia: Usos e Perspectivas. Revista Brasileira de Ciências da Amazônia, 1: 213 231.

SILVA, E. R.; BARROS, D. R.; KINUPP, V. F.; ALFAIA, S. S.; ALVES, M. I. C.; COIMBRA, A.B. Isoporização em Cará (Dioscorea Altissima Lam.). Cadernos de Agroecologia, vol. 10, $\mathrm{n}^{\circ}$ 3, Amazonas, 2016. Disponível em: /\%//http://aba-agro ecologia.org.br/ revistas/index.php/cad/article/view/17960/11816. 